\title{
Toxicity Study of a Novel Oral Iron Chelator: 1-(N-Acetyl-6-Aminohexyl)-3 Hydroxy-2-Methylpyridin-4-One (CM1) in Transgenic $\beta$-Thalassemia Mice
}

Nittaya Chansiw ${ }^{1}$, Kanjana Pangjit ${ }^{1,2}$, Chada Phisalaphong ${ }^{3}$, Suthat Fucharoen ${ }^{4}$, Patricia Evans ${ }^{5}$, John B Porter ${ }^{5}$ and Somdet Srichairatanakool ${ }^{1 *}$

${ }^{1}$ Department of Biochemistry, Faculty of Medicine, Chiang Mai University, Thailand

${ }^{2}$ College of Medicine and Public Health, Ubon Ratchathani University, Thailand

3 Institute of Research and Development, Government Pharmaceutical Organization, Ministry of Public Health, Bangkok 10400, Thailand

${ }^{4}$ Thalassemia Research Center, Institute of Molecular Bioscience, Mahidol University Salaya Campus, Thailand

${ }^{5}$ Department of Haematology, UCL Cancer Institute, Paul O' Gorman Building, Huntley Street, United Kingdom

\begin{abstract}
Deferiprone (DFP) (MW=139 Da, Kpart=0.11) is an effective iron chelator used for the treatment of iron overload in thalassemia patients, but the drug is not free from side effects. We have synthesized a novel oral bidentate iron chelator, 1-(N-acetyl-6-aminohexyl)-3-hydroxypyridin-4-one (CM1) (MW=256 Da, Kpart=0.53), which is an analogue of DFP. This compound is more lipophilic than DFP and can bind iron efficiently. Our current results have demonstrated that CM1 reduced iron-induced redox damage and decreased levels of the intracellular iron pool (LIP) in cultured hepatocytes, effectively. However, the toxicity of CM1 remains largely unknown. The aim of this study was to therefore examine the toxicity of $\mathrm{CM} 1$ treatment in an animal model under normal and iron overload conditions. To induce iron overload, transgenic ß-thalassemia (BKO) mice were fed with a $0.2 \%(\mathrm{w} / \mathrm{w})$ ferrocene-supplemented diet (Fe diet) for 240 days. The mice received three doses of CM1 orally $(50,100$ and $200 \mathrm{mg} / \mathrm{kg})$, every day for 180 days. Blood was collected from the tail vein every 45 days during treatment for the measurement of hemoglobin $(\mathrm{Hb})$ levels, white blood cells (WBC) and platelet numbers. We also determined the activities of alanine aminotransferase (ALT), aspartate aminotransferase (AST) and alkaline phosphatase (ALP), which are markers of liver damage. Treatment with CM1 at the assigned doses did not markedly alter the numbers of WBC and the platelets, and the $\mathrm{Hb}$ level in BKO mice fed with either $\mathrm{N}$ diet or Fe diet. Importantly, all the treatments slightly increased the activities of plasma AST, ALT and ALP in BKO mice after 150 days. Nonetheless, hematoxylin and eosin staining results did not show abnormal morphological changes of the spleen, liver and heart tissues. The results imply that CM1 may not be toxic to bone marrow cells and liver cell function in BKO mice under normal and iron overload conditions.
\end{abstract}

Keywords: 3-Hydroxypyridinone; Iron chelator; Iron overload; Toxicity; ß-thalassemia

\section{Introduction}

Iron is absolutely essential to living cells and plays many important roles in energy production, oxygen transport and DNA synthesis. Although iron is crucial for cells, an excess of iron is toxic [1]. In thalassemia patients, multiple blood transfusions and abnormal iron absorption cause iron overload and accumulation in tissues and organs [2]. Consequently, reactive oxygen species (ROS) are produced from the iron-catalyzed Fenton reaction, causing oxidative tissue damage and organ dysfunctions $[3,4]$. Iron chelation therapy is required, not just to restore iron balance, but also to reduce toxic iron species. Iron chelators, such as desferrioxamine (DFO), deferiprone (DFP) and deferasirox (DFX), are available for clinical use, but these compounds still have many side effects and drawbacks. DFO is not orally active [5], DFX is associated with renal toxicity in some patients [6], and DFP can have side effects, including nausea, vomiting, gastrointestinal tract disturbances, leucopenia, thrombocytopenia and zinc deficiency [7]. We have successfully synthesized a novel oral bidentate iron chelator, 1-(N-acetyl-6-aminohexyl)-3-hydroxypyridin-4-one (CM1). It is DFP analogue and is more lipophilic than DFP. In a previous study, we found that CM1 was able to bind both ferric and ferrous ions, and was also able to chelate plasma non-transferrin bound iron (NTBI), efficiently [8]. Moreover, we found that the CM1 was not toxic to peripheral blood mononuclear cells and liver cells in an in vitro study [9]. However, the toxicity of CM1 in the in vivo study remains largely unknown. In this study, we examined the toxicity of CM1 on the liver and peripheral blood cells in transgenic $ß$-thalassemia mice under normal and iron overload conditions.

\section{Materials and Methods}

In the experimental design of this study, the heterozygous
B-thalassemia knockout (BKO, mu?thal-3/+) mice strain $\mathrm{C} 57 \mathrm{BL} / 6$, as well as the weight $20 \pm 5 \mathrm{~g}$, were recorded. During the entire study period, the mice were housed in hygienic cages in a controlled environment $(12$ hours light/dark cycle and $25 \pm 3^{\circ} \mathrm{C}$ ). The mice were randomized into 2 groups (40 males and 40 females for each group): one group were fed a normal pellet diet ( $\mathrm{N}$ diet), and the other group were fed a $0.2 \%(\mathrm{w} / \mathrm{w})$ ferrocene-supplemented pellet diet (Fe diet) to induce iron overload, over 240 days [10]. Both groups of the mice were then segregated as follows: ten mice from each group received the deionized water (DI) placebo, and thirty mice from each group received 1-(N-acetyl-6aminohexyl)-3 hydroxypyridin-4-one (CM1), and were synthesized by our group [11]. The mice received CM1 at the doses of 50, 100 and $200 \mathrm{mg} / \mathrm{kg}$ (10 mice each) orally for 180 days. Clinical observations were performed daily and body weight was recorded weekly. Tail vein blood was collected for prior to beginning the study, at 60 days, and at every 45 days during treatment. The samples were investigated for the hematological and biochemical parameters. Additionally, four organs of the mice, including the heart, liver, kidneys and spleen were collected after termination to perform the pathological examination.

*Corresponding author: Somdet Srichairattanakool, Department of Biochemistry, Faculty of Medicine, Chiang Mai University, Thailand, E-mail: ssrichai@med.cmu.ac.th

Received July 08, 2013; Accepted August 01, 2013; Published August 06, 2013

Citation: Chansiw N, Pangjit K, Phisalaphong C, Fucharoen S, Evans P, et al (2013) Toxicity Study of a Novel Oral Iron Chelator: 1-(N-Acetyl-6-Aminohexyl)-3 Hydroxy-2-Methylpyridin-4-One (CM1) in Transgenic ß-Thalassemia Mice. Vitam Miner 2: 116

Copyright: ( $) 2013$ Chansiw N, et al. This is an open-access article distributed under the terms of the Creative Commons Attribution License, which permits unrestricted use, distribution, and reproduction in any medium, provided the original author and source are credited. 


\section{Hematological parameters}

The samples were drawn into a heparin-containing capillary tube. Whole blood was taken to measure hemoglobin concentrations following the cyanmethemoglobin method [12]. White blood cells and platelet numbers were diluted with WBC (1:50) and Platelet (1:200) diluting solution (Biotech Co. Ltd., Bangkok, Thailand), respectively, and were counted with a hematocytometer under microscopy.

\section{Biochemical parameters}

Blood samples were centrifuged at 3,000 $\mathrm{g}$ for $10 \mathrm{~min}$ to separate the plasma. Biochemical parameters, including aspartate aminotransferase (AST), alanine aminotransferase (ALT) and alkaline phosphatase (ALP) activities, were assessed using enzyme assay kits (Biotech Co. Ltd., Bangkok, Thailand) [13,14].

\section{Pathological examination}

At the termination of the study, all groups were weighed and sacrificed for the collection of their organs, including the heart, liver, spleen and kidneys. After that each organ was weighed, parts of the heart, liver and spleen were fixed in $10 \%$ neutral buffered formalin. Then, the tissues were dehydrated with a graded series of ethyl alcohol, embedded in paraffin wax and finally sectioned and stained with Hematoxylin \& Eosin (H\&E) dye. The stained slides were examined under a light microscope by an expert pathologist, and were then photographed with a digital camera.

\section{Statistical analysis}

The results were expressed as Means + SEM. Statistical significance was determined using a one-way analysis of variance (ANOVA), in which $\mathrm{p}<0.05$ was considered significant.

\section{Results}

\section{Clinical observations and body weight}

No clinical sign of toxicity was observed in the B-thalassemia mice that were fed with $\mathrm{N}$ and $\mathrm{Fe}$ diets at all doses of CM1, over 240 days. Body weight was not different in the placebo and the CM1 treated groups, however, it was slightly increased in all groups of mice, and body weight was similar in both the $\mathrm{N}$ and $\mathrm{Fe}$ fed groups.

\section{Impact of CM1 on haematological parameters}

In the previous in vitro study, we found that CM1 is an effective iron chelator that is non-toxic to peripheral blood mononuclear cells (PBMC). However, the toxicity of CM1 in the animal model is unknown. To investigate whether or not CM1 has toxicity to the peripheral cells in the animal study; $ß$-thalassemia mice were fed with an $\mathrm{N}$ diet or $\mathrm{Fe}$ diet, and the dietary routine was then intervened with DI water for the placebo group and CM1 at 50, 100 and $200 \mathrm{mg} / \mathrm{kg}$ for the test groups. The results showed that haemoglobin levels were not different between the placebo, and/or normally treated mice (Table 1 ), and the iron-loaded groups (Table 2). That could imply that the type of food and CM1 concentrations were not affected over the 180day period. We also found that the chelator did not produce a drop in numbers of the WBC and the platelets (Table 1 and 3), there was no difference between the placebo and the treated groups and results were similar among the mice fed with both the $\mathrm{N}$ and Fe diets. The experimental stage in this study demonstrated that CM1 was not toxic to peripheral blood cells under normal and iron-overload conditions.

\section{Effect of CM1 on biochemical parameters}

To investigate whether CM1 damaged hepatocyte cells in the in vivo study, we examined marker enzymes for liver damage, including AST, ALT and ALP using enzymes test kits based on spectrophotometric analysis. The results showed that all of the enzymes in both the placebo and the treated mice were not found to be significantly different, under both dietary conditions. There were also no dose-related effects. In both the normal diet and iron rich consumption groups, AST was slightly increased after 60 days and reached a peak at 150 days, before dropping 45 days later (Table 4). However, the trend was not shown in the ALT and ALP measurements. ALT and ALP slightly increased after 60 days. Nevertheless, these changes were still not dose-dependent (Table 2 and 4).

\section{Organ weights, organ/body weight ratio and histopathological examination}

After the termination of the study, the mice were collected and

\begin{tabular}{|c|c|c|c|c|c|c|c|c|c|c|c|c|}
\hline \multicolumn{5}{|c|}{ Hemoglobin concentration (g/dl) } & \multicolumn{4}{|c|}{ WBC numbers (109/I) } & \multicolumn{4}{|c|}{ Platelet numbers $(109 / 1)$} \\
\hline \multicolumn{5}{|c|}{ Treatment } & \multicolumn{4}{|c|}{ Treatment } & \multicolumn{4}{|c|}{ Treatment } \\
\hline Days & DI & $\begin{array}{c}\mathrm{CM} 1 \\
(50 \mathrm{mg} / \mathrm{kg})\end{array}$ & $\begin{array}{c}\text { CM1 } \\
(100 \mathrm{mg} / \mathrm{kg})\end{array}$ & $\begin{array}{c}\text { CM1 } \\
(200 \mathrm{mg} / \mathrm{kg})\end{array}$ & DI & $\begin{array}{c}\mathrm{CM} 1 \\
(50 \mathrm{mg} / \mathrm{kg})\end{array}$ & $\begin{array}{c}\text { CM1 } \\
(100 \mathrm{mg} / \mathrm{kg})\end{array}$ & $\begin{array}{c}\text { CM1 } \\
(200 \mathrm{mg} / \mathrm{kg})\end{array}$ & DI & $\begin{array}{c}\mathrm{CM} 1 \\
(50 \mathrm{mg} / \mathrm{kg})\end{array}$ & $\begin{array}{c}\text { CM1 } \\
(100 \mathrm{mg} / \mathrm{kg})\end{array}$ & $\begin{array}{c}\text { CM1 } \\
(200 \mathrm{mg} / \mathrm{kg})\end{array}$ \\
\hline 0 & $10.25 \pm 0.89$ & $11.35 \pm 1.28$ & $10.73 \pm 0.36$ & $10.05 \pm 0.42$ & $.89 \pm 2.30$ & $4.65 \pm 1.90$ & $5.83 \pm 1.38$ & $3.43 \pm 0.68$ & $360.0 \pm 39.8$ & $422.0 \pm 62.2$ & $385.6 \pm 43.4$ & $411.2 \pm 40.0$ \\
\hline 60 & $10.79 \pm 0.50$ & $12.29 \pm 0.90$ & $10.82 \pm 0.38$ & $10.66 \pm 0.26$ & $7.57 \pm 1.77$ & $6.67 \pm 2.72$ & $6.58 \pm 1.67$ & $6.07 \pm 1.79$ & $425.6 \pm 42.2$ & $424.0 \pm 43.5$ & $376.0 \pm 18.4$ & $448.8 \pm 52.5$ \\
\hline 105 & $11.02 \pm 0.56$ & $11.53 \pm 0.87$ & $10.73 \pm 0.35$ & $10.33 \pm 0.39$ & $7.60 \pm 0.86$ & $4.98 \pm 2.03$ & $7.56 \pm 1.48$ & $8.57 \pm 2.20$ & $249.5 \pm 88.5$ & $472.0 \pm 145.2$ & $248.0 \pm 69.2$ & $286.4 \pm 73.6$ \\
\hline 150 & $10.14 \pm 0.44$ & $9.82 \pm 0.65$ & $9.05 \pm 1.18$ & $10.92 \pm 0.67$ & $8.62 \pm 2.14$ & $4.74 \pm 2.12$ & $10.54 \pm 1.90$ & $11.10 \pm 3.60$ & $445.0 \pm 199.9$ & $322.4 \pm 51.3$ & $265.5 \pm 72.4$ & $516.8 \pm 265.0$ \\
\hline 195 & $9.34 \pm 0.87$ & $10.39 \pm 0.55$ & $11.10 \pm 1.30$ & $10.20 \pm 1.11$ & $12.7 \pm 2.64$ & $5.63 \pm 2.52$ & $11.15 \pm 1.44$ & $14.42 \pm 2.96$ & $609.0 \pm 302.1$ & $534.0 \pm 189.8$ & $416.5 \pm 126.3$ & $486.5 \pm 281.6$ \\
\hline 240 & $7.95 \pm 0.51$ & $9.10 \pm 0.64$ & $8.56 \pm 0.61$ & $8.94 \pm 0.72$ & $6.25 \pm 0.88$ & $2.58 \pm 1.15$ & $7.05 \pm 1.56$ & $7.33 \pm 1.74$ & $375.5 \pm 78.2$ & $433.2 \pm 84.3$ & $373.5 \pm 98.4$ & $490.6 \pm 254.7$ \\
\hline
\end{tabular}

Table 1: Effect of CM1 treatment on hematological parameters in $\beta$-thalassemia mice $(B K O)$ fed with $N$ diet. Data were expressed as mean + SEM

\begin{tabular}{|c|c|c|c|c|c|c|c|c|c|c|c|c|}
\hline \multirow{2}{*}{\multicolumn{5}{|c|}{ Hemoglobin concentration (g/dl) }} & \multirow{2}{*}{\multicolumn{4}{|c|}{ WBC numbers (109/l) }} & \multicolumn{4}{|c|}{ Platelet numbers (109/l) } \\
\hline & & & & & & & & & & Trea & atment & \\
\hline Days & DI & $\begin{array}{c}\mathrm{CM} 1 \\
(50 \mathrm{mg} / \mathrm{kg})\end{array}$ & $\begin{array}{c}\text { CM1 } \\
(100 \mathrm{mg} / \mathrm{kg})\end{array}$ & $\begin{array}{c}\text { CM1 } \\
(200 \mathrm{mg} / \mathrm{kg})\end{array}$ & DI & $\begin{array}{c}\mathrm{CM} 1 \\
(50 \mathrm{mg} / \mathrm{kg})\end{array}$ & $\begin{array}{c}\text { CM1 } \\
(100 \mathrm{mg} / \mathrm{kg})\end{array}$ & $\begin{array}{c}\text { CM1 } \\
(200 \mathrm{mg} / \mathrm{kg})\end{array}$ & DI & $\begin{array}{c}\mathrm{CM} 1 \\
(50 \mathrm{mg} / \mathrm{kg})\end{array}$ & $\begin{array}{c}\text { CM1 } \\
(100 \mathrm{mg} / \mathrm{kg})\end{array}$ & $\begin{array}{c}\text { CM1 } \\
(200 \mathrm{mg} / \mathrm{kg})\end{array}$ \\
\hline 0 & $10.49 \pm 0.77$ & $11.28 \pm 0.33$ & $10.94 \pm 0.40$ & $10.87 \pm 0.36$ & $5.05 \pm 0.58$ & $3.43 \pm 0.39$ & $3.70 \pm 0.61$ & $3.31 \pm 0.54$ & $425.6 \pm 39.8$ & $387.3 \pm 52.2$ & $437.7 \pm 35.9$ & $387.4 \pm 22.0$ \\
\hline 60 & $11.44 \pm 0.88$ & $11.72 \pm 0.63$ & $12.21 \pm 0.75$ & $11.76 \pm 0.39$ & $10.22 \pm 2.82$ & $11.46 \pm 3.36$ & $11.73 \pm 2.74$ & $12.33 \pm 2.19$ & $395.2 \pm 35.7$ & $443.3 \pm 51.3$ & $363.3 \pm 19.5$ & $405.3 \pm 36.7$ \\
\hline 105 & $9.99 \pm 0.70$ & $11.90 \pm 0.82$ & $11.20 \pm 0.53$ & $11.53 \pm 0.58$ & $17.90 \pm 2.92$ & $17.67 \pm 1.91$ & $13.99 \pm 2.54$ & $17.65 \pm 3.74$ & $259.2 \pm 59.2$ & $290.0 \pm 69.1$ & $234.0 \pm 79.2$ & $202.0 \pm 47.2$ \\
\hline 150 & $10.00 \pm 0.57$ & $10.87 \pm 0.68$ & $10.74 \pm 0.57$ & $11.67 \pm 0.78$ & $23.08 \pm 5.10$ & $26.74 \pm 4.72$ & $17.18 \pm 3.45$ & $30.24 \pm 5.82$ & $218.0 \pm 32.5$ & $258.6 \pm 59.0$ & $320.6 \pm 105.7$ & $170.3 \pm 19.84$ \\
\hline 195 & $10.77 \pm 1.18$ & $9.72 \pm 0.95$ & $10.64 \pm 0.57$ & $10.38 \pm 1.00$ & $16.83 \pm 2.31$ & $30.73 \pm 2.44$ & $31.55 \pm 2.87$ & $37.62 \pm 3.11^{*}$ & $332.0 \pm 53.3$ & $407.6 \pm 129.8$ & $335.0 \pm 157.1$ & $364.0 \pm 150.36$ \\
\hline 240 & $8.61 \pm 0.80$ & $9.33 \pm 0.38$ & $8.63 \pm 0.51$ & $7.94 \pm 0.44$ & $13.65 \pm 2.96$ & $25.82 \pm 6.06$ & $23.25 \pm 7.83$ & $22.28 \pm 5.30$ & $408.0 \pm 28.6$ & $552.8 \pm 149.8$ & $398.5 \pm 54.2$ & $425.2 \pm 66.3$ \\
\hline
\end{tabular}

Table 2: Effect of CM1 treatment on hematological parameters in $\beta$-thalassemia mice (BKO) fed with Fe diet. Data were expressed as mean + SEM. 
Citation: Chansiw N, Pangjit K, Phisalaphong C, Fucharoen S, Evans P, et al. (2013) Toxicity Study of a Novel Oral Iron Chelator: 1-(N-Acetyl-6Aminohexyl)-3 Hydroxy-2-Methylpyridin-4-One (CM1) in Transgenic ß-Thalassemia Mice. Vitam Miner 2: 116.

Page 3 of 6

their organs, including the heart, liver, kidneys and spleen were excised and weighed. Organ weights and organ/body weight ratios are shown in Tables 5-8. No significant differences were observed between the placebo and the CM1 treatment groups, in both normal and iron loading conditions. Results of histopathological examination are shown in Figure 2. For the mice treated with DI water and CM1 in the $\mathrm{N}$ and Fe diet fed groups, the heart tissue did not show any significant pathological change. The liver tissue showed a moderate degree of spotty necrosis, particularly in the portal area and the neutrophils, and lymphocytes were predominantly associated with numerous hemosiderin-laden macrophages in hepatic sinusoids. The spleen tissue presented numerous hemosiderin-laden macrophages in both the red pulp and white pulp, particularly in the Fe diet fed group. The results suggested that CM1 was not toxic to tissue organs, including the heart, liver and spleen.

\begin{tabular}{|c|c|c|c|c|c|c|c|c|c|c|c|c|}
\hline \multicolumn{5}{|c|}{ AST (U/L) } & \multicolumn{4}{|c|}{ ALT (U/L) } & \multicolumn{4}{|c|}{ ALP (U/L) } \\
\hline \multicolumn{5}{|c|}{ Treatment } & \multicolumn{4}{|c|}{ Treatment } & \multicolumn{4}{|c|}{ Treatment } \\
\hline Days & DI & $\begin{array}{c}\text { CM1 } \\
(50 \mathrm{mg} / \mathrm{kg})\end{array}$ & $\begin{array}{c}\mathrm{CM} 1 \\
(100 \mathrm{mg} / \mathrm{kg})\end{array}$ & $\begin{array}{c}\text { CM1 } \\
(200 \mathrm{mg} / \\
\mathrm{kg})\end{array}$ & DI & $\begin{array}{c}\mathrm{CM} 1 \\
(50 \mathrm{mg} / \mathrm{kg})\end{array}$ & $\begin{array}{c}\text { CM1 } \\
(100 \mathrm{mg} / \\
\mathrm{kg})\end{array}$ & $\begin{array}{c}\text { CM1 } \\
(200 \mathrm{mg} / \\
\mathrm{kg})\end{array}$ & DI & $\begin{array}{c}\text { CM1 } \\
(50 \mathrm{mg} / \mathrm{kg})\end{array}$ & $\begin{array}{c}\text { CM1 } \\
(100 \mathrm{mg} / \mathrm{kg})\end{array}$ & $\begin{array}{c}\text { CM1 } \\
(200 \mathrm{mg} / \mathrm{kg})\end{array}$ \\
\hline 0 & $16 \pm 5$ & $26 \pm 7$ & $14 \pm 4$ & $14 \pm 4$ & $15 \pm 2$ & $17 \pm 3$ & $9 \pm 4$ & $12 \pm 3$ & $26 \pm 4$ & $25 \pm 4$ & $18 \pm 6$ & $20 \pm 7$ \\
\hline 60 & $38 \pm 4$ & $39 \pm 4$ & $20 \pm 7$ & $27 \pm 9$ & $35 \pm 9$ & $49 \pm 17$ & $39 \pm 24$ & $54 \pm 28$ & $24 \pm 2$ & $25 \pm 2$ & $16 \pm 6$ & $16 \pm 5$ \\
\hline 105 & $38 \pm 7$ & $39 \pm 8$ & $25 \pm 9$ & $17 \pm 5$ & $31 \pm 3$ & $36 \pm 6$ & $20 \pm 6$ & $17 \pm 5$ & $36 \pm 6$ & $40 \pm 3$ & $24 \pm 9$ & $22 \pm 5$ \\
\hline 150 & $111 \pm 37^{*}$ & $85 \pm 30$ & $77 \pm 32$ & $71 \pm 26^{*}$ & $19 \pm 4$ & $28 \pm 10$ & $19 \pm 5$ & $32 \pm 14$ & $34 \pm 4$ & $28 \pm 3$ & $21 \pm 9$ & $31 \pm 16$ \\
\hline 195 & $64 \pm 9$ & $53 \pm 4$ & $36 \pm 15$ & $40 \pm 12$ & $41 \pm 20$ & $62 \pm 14$ & $36 \pm 19$ & $53 \pm 17$ & $41 \pm 8$ & $34 \pm 5$ & $27 \pm 9$ & $23 \pm 8$ \\
\hline 240 & $56 \pm 30$ & $100 \pm 36$ & $67 \pm 41$ & $65 \pm 31$ & $13 \pm 12$ & $18 \pm 10$ & $14 \pm 11$ & $18 \pm 10$ & $39 \pm 5$ & $40 \pm 4$ & $27 \pm 10$ & $25 \pm 9$ \\
\hline
\end{tabular}

Table 3: Effect of CM1 treatment on biochemical parameters in $\beta$-thalassemia mice $(B K O)$ fed with $N$ diet. Data were expressed as mean $+\mathrm{SEM}$.

\begin{tabular}{|c|c|c|c|c|c|c|c|c|c|c|c|c|}
\hline \multicolumn{5}{|c|}{ AST (U/L) } & \multicolumn{4}{|c|}{ ALT (U/L) } & \multicolumn{4}{|c|}{ ALP (U/L) } \\
\hline \multicolumn{5}{|c|}{ Treatment } & \multicolumn{4}{|c|}{ Treatment } & \multicolumn{4}{|c|}{ Treatment } \\
\hline Days & DI & $\begin{array}{c}\text { CM1 } \\
(50 \mathrm{mg} / \mathrm{kg})\end{array}$ & $\begin{array}{c}\text { CM1 } \\
(100 \mathrm{mg} / \mathrm{kg})\end{array}$ & $\begin{array}{c}\text { CM1 } \\
(200 \mathrm{mg} / \mathrm{kg})\end{array}$ & DI & $\begin{array}{c}\mathrm{CM} 1 \\
(50 \mathrm{mg} / \mathrm{kg})\end{array}$ & $\begin{array}{c}\mathrm{CM} 1 \\
(100 \mathrm{mg} / \mathrm{kg})\end{array}$ & $\begin{array}{c}\mathrm{CM} 1 \\
(200 \mathrm{mg} / \mathrm{kg})\end{array}$ & DI & $\begin{array}{c}\text { CM1 } \\
(50 \mathrm{mg} / \mathrm{kg})\end{array}$ & $\begin{array}{c}\text { CM1 } \\
(100 \mathrm{mg} / \mathrm{kg})\end{array}$ & $\begin{array}{c}\mathrm{CM} 1 \\
(200 \mathrm{mg} / \mathrm{kg})\end{array}$ \\
\hline 0 & $43 \pm 10$ & $22 \pm 5$ & $29 \pm 6$ & $24 \pm 4$ & $17 \pm 1$ & $17 \pm 2$ & $16 \pm 3$ & $15 \pm 3$ & $32 \pm 5$ & $28 \pm 3$ & $26 \pm 4$ & $35 \pm 5$ \\
\hline 60 & $65 \pm 2$ & $78 \pm 4$ & $74 \pm 8$ & $83 \pm 6^{*}$ & $65 \pm 7$ & $74 \pm 7^{*}$ & $79 \pm 12$ & $79 \pm 14$ & $33 \pm 2$ & $28 \pm 2$ & $28 \pm 3$ & $25 \pm 2$ \\
\hline 105 & $64 \pm 15$ & $72 \pm 13$ & $66 \pm 12$ & $101 \pm 15^{\star}$ & $64 \pm 14$ & $85 \pm 13^{*}$ & $80 \pm 8$ & $114 \pm 11$ & $42 \pm 5$ & $50 \pm 8$ & $50 \pm 7$ & $44 \pm 6$ \\
\hline 150 & $157 \pm 39^{*}$ & $163 \pm 44^{*}$ & $159 \pm 40^{*}$ & $174 \pm 27^{*}$ & $39 \pm 11$ & $53 \pm 15$ & $49 \pm 17$ & $62 \pm 14$ & $36 \pm 4$ & $32 \pm 2$ & $32 \pm 5$ & $31 \pm 5$ \\
\hline 195 & $100 \pm 6$ & $111 \pm 12^{*}$ & $109 \pm 5$ & $108 \pm 12^{*}$ & $39 \pm 12$ & $60 \pm 17$ & $60 \pm 19$ & $54 \pm 14$ & $72 \pm 13^{*}$ & $58 \pm 10$ & $74 \pm 15^{*}$ & $54 \pm 9$ \\
\hline 240 & $94 \pm 27$ & $80 \pm 22$ & $88 \pm 34$ & $57 \pm 12$ & $113 \pm 57$ & $69 \pm 22$ & $81 \pm 48$ & $114 \pm 62$ & $57 \pm 7$ & $49 \pm 6$ & $62 \pm 10^{*}$ & $51 \pm 9$ \\
\hline
\end{tabular}

Table 4: Effect of CM1 treatment on biochemical parameters in $\beta$-thalassemia mice (BKO) fed with Fe diet. Data were expressed as means + SEM.

\begin{tabular}{|c|c|c|c|c|}
\hline \multicolumn{5}{|c|}{ Treatment } \\
\hline \multirow{2}{*}{ Parameter } & \multirow{2}{*}{ DI } & CM1 & CM1 & CM1 \\
\hline & & $(50 \mathrm{mg} / \mathrm{kg})$ & $(100 \mathrm{mg} / \mathrm{kg})$ & $(200 \mathrm{mg} / \mathrm{kg})$ \\
\hline \multicolumn{5}{|c|}{ Organ weight (g) } \\
\hline Heart & $0.19 \pm 0.005$ & $0.17 \pm 0.01$ & $0.22 \pm 0.02$ & $0.17 \pm 0.01$ \\
\hline Liver & $1.89 \pm 0.04$ & $1.69 \pm 0.11$ & $1.85 \pm 0.08$ & $1.51 \pm 0.11$ \\
\hline Kidneys & $0.46 \pm 0.02$ & $0.44 \pm 0.03$ & $0.45 \pm 0.01$ & $0.42 \pm 0.02$ \\
\hline Spleen & $0.26 \pm 0.03$ & $0.29 \pm 0.06$ & $0.50 \pm 0.16$ & $0.28 \pm 0.01$ \\
\hline \multicolumn{5}{|c|}{ Organ weight index } \\
\hline Heart & $0.0060 \pm 0.0002$ & $0.0059 \pm 0.0005$ & $0.0074 \pm 0.0007$ & $0.0063 \pm 0.0005$ \\
\hline Liver & $0.0603 \pm 0.0009$ & $0.0560 \pm 0.0030$ & $0.0620 \pm 0.0030$ & $0.0540 \pm 0.0020$ \\
\hline Kidneys & $0.0145 \pm 0.0006$ & $0.0147 \pm 0.0008$ & $0.0150 \pm 0.0002$ & $0.0150 \pm 0.0010$ \\
\hline Spleen & $0.0082 \pm 0.0009$ & $0.0100 \pm 0.0020$ & $0.0170 \pm 0.0050$ & $0.0102 \pm 0.0009$ \\
\hline
\end{tabular}

Table 5: Organ weight and organ weight index in male $\beta$-thalassemic mice (BKO) fed with an $\mathrm{N}$ diet and treated with CM1 at the assigned doses for 180 days. Data were expressed as means + SEM.

\begin{tabular}{|c|c|c|c|c|}
\hline \multicolumn{5}{|c|}{ Treatment } \\
\hline Parameter & $\mathrm{DI}$ & CM1 (50 mg/kg) & CM1 (100 mg/kg) & CM1 $(200 \mathrm{mg} / \mathrm{kg})$ \\
\hline \multicolumn{5}{|c|}{ Mean weight (g) } \\
\hline Heart & $0.139 \pm 0.007$ & $0.136 \pm 0.005$ & $0.133 \pm 0.009$ & $0.14 \pm 0.01$ \\
\hline Liver & $1.34 \pm 0.06$ & $1.35 \pm 0.10$ & $1.31 \pm 0.04$ & $1.33 \pm 0.04$ \\
\hline Kidneys & $0.32 \pm 0.02$ & $0.33 \pm 0.02$ & $0.32 \pm 0.02$ & $0.35 \pm 0.02$ \\
\hline Spleen & $0.35 \pm 0.02$ & $0.38 \pm 0.03$ & $0.37 \pm 0.06$ & $0.36 \pm 0.02$ \\
\hline \multicolumn{5}{|c|}{ Mean organ to terminal body weight ratios } \\
\hline Heart & $0.0061 \pm 0.0003$ & $0.0057 \pm 0.0002$ & $0.0056 \pm 0.0005$ & $0.0060 \pm 0.0006$ \\
\hline Liver & $0.058 \pm 0.001$ & $0.056 \pm 0.003$ & $0.055 \pm 0.002$ & $0.0569 \pm 0.0003$ \\
\hline Kidneys & $0.014 \pm 0.001$ & $0.0139 \pm 0.0007$ & $0.014 \pm 0.001$ & $0.0151 \pm 0.0007$ \\
\hline Spleen & $0.015 \pm 0.002$ & $0.016 \pm 0.001$ & $0.016 \pm 0.002$ & $0.015 \pm 0.001$ \\
\hline
\end{tabular}

Table 6: Organ weight and organ weight index in female mice treated with CM1 and fed with normal diets for 180 days. Data were expressed as means + SEM. 
Citation: Chansiw N, Pangjit K, Phisalaphong C, Fucharoen S, Evans P, et al. (2013) Toxicity Study of a Novel Oral Iron Chelator: 1-(N-Acetyl-6Aminohexyl)-3 Hydroxy-2-Methylpyridin-4-One (CM1) in Transgenic ß-Thalassemia Mice. Vitam Miner 2: 116.

Page 4 of 6

\begin{tabular}{|c|c|c|c|c|}
\hline \multicolumn{5}{|c|}{ Treatment } \\
\hline Parameter & DI & $\begin{array}{c}\mathrm{CM} 1 \\
(50 \mathrm{mg} / \mathrm{kg})\end{array}$ & $\begin{array}{c}\text { CM1 } \\
(100 \mathrm{mg} / \mathrm{kg})\end{array}$ & $\begin{array}{c}\text { CM1 } \\
(200 \mathrm{mg} / \mathrm{kg})\end{array}$ \\
\hline \multicolumn{5}{|c|}{ Organ weight (g) } \\
\hline Heart & $0.140 \pm 0.003$ & $0.14 \pm 0.01$ & $0.13 \pm 0.005$ & $0.17 \pm 0.02$ \\
\hline Liver & $3.47 \pm 0.12$ & $3.22 \pm 0.38$ & $3.34 \pm 0.11$ & $3.84 \pm 0.32$ \\
\hline Kidneys & $0.31 \pm 0.04$ & $0.290 \pm 0.006$ & $0.29 \pm 0.02$ & $0.33 \pm 0.02$ \\
\hline Spleen & $0.37 \pm 0.04$ & $0.299 \pm 0.005$ & $0.40 \pm 0.01$ & $0.42 \pm 0.04$ \\
\hline \multicolumn{5}{|c|}{ Organ weight index } \\
\hline Heart & $0.0058 \pm 0.0003$ & $0.006 \pm 0.003$ & $0.0060 \pm 0.0002$ & $0.0066 \pm 0.0008$ \\
\hline Liver & $0.15 \pm 0.01$ & $0.14 \pm 0.13$ & $0.144 \pm 0.0 .007$ & $0.151 \pm 0.008$ \\
\hline Kidneys & $0.013 \pm 0.001$ & $0.013 \pm 0.005$ & $0.0128 \pm 0.0005$ & $0.0131 \pm 0.0006$ \\
\hline Spleen & $0.015 \pm 0.001$ & $0.013 \pm 0.003$ & $0.0176 \pm 0.0009$ & $0.017 \pm 0.001$ \\
\hline
\end{tabular}

Table 7: Organ weight and organ weight index in male mice treated with $\mathrm{CM} 1$ and fed with Fe diet for 180 days. Data were expressed as means $+\mathrm{SEM}$.

\begin{tabular}{|c|c|c|c|c|}
\hline \multicolumn{5}{|c|}{ Treatment } \\
\hline Parameter & DI & CM1 (50 mg/kg) & CM1 (100 mg/kg) & CM1 $(200 \mathrm{mg} / \mathrm{kg})$ \\
\hline \multicolumn{5}{|c|}{ Organ weight (g) } \\
\hline Heart & $0.127 \pm 0.007$ & $0.133 \pm 0.008$ & $0.12 \pm 0.02$ & $0.14 \pm 0.01$ \\
\hline Liver & $2.78 \pm 0.32$ & $2.49 \pm 0.08$ & $2.91 \pm 0.23$ & $2.62 \pm 0.14$ \\
\hline Kidneys & $0.28 \pm 0.02$ & $0.263 \pm 0.009$ & $0.27 \pm 0.02$ & $0.30 \pm 0.02$ \\
\hline Spleen & $0.42 \pm 0.03$ & $0.37 \pm 0.01$ & $0.38 \pm 0.06$ & $0.39 \pm 0.01$ \\
\hline \multicolumn{5}{|c|}{ Organ weight index } \\
\hline Heart & $0.0064 \pm 0.0005$ & $0.0066 \pm 0.0004$ & $0.0061 \pm 0.0007$ & $0.0066 \pm 0.0006$ \\
\hline Liver & $0.14 \pm 0.01$ & $0.124 \pm 0.008$ & $0.144 \pm 0.007$ & $0.129 \pm 0.005$ \\
\hline Kidneys & $0.0139 \pm 0.0009$ & $0.0131 \pm 0.0004$ & $0.0139 \pm 0.0008$ & $0.0146 \pm 0.0002$ \\
\hline Spleen & $0.021 \pm 0.001$ & $0.0186 \pm 0.0007$ & $0.019 \pm 0.002$ & $0.0194 \pm 0.0009$ \\
\hline
\end{tabular}

Table 8: Organ weight and organ weight index in female mice treated with CM1 and fed with Fe diet for 180 days. Data were expressed as means + SEM.

Male/N diet

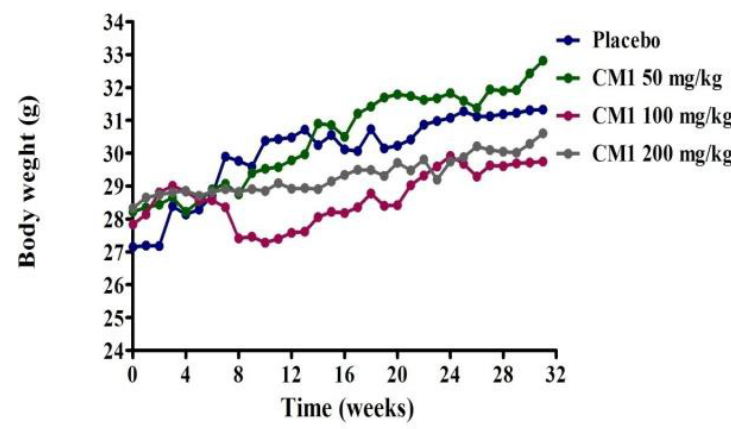

Male/Fe diet

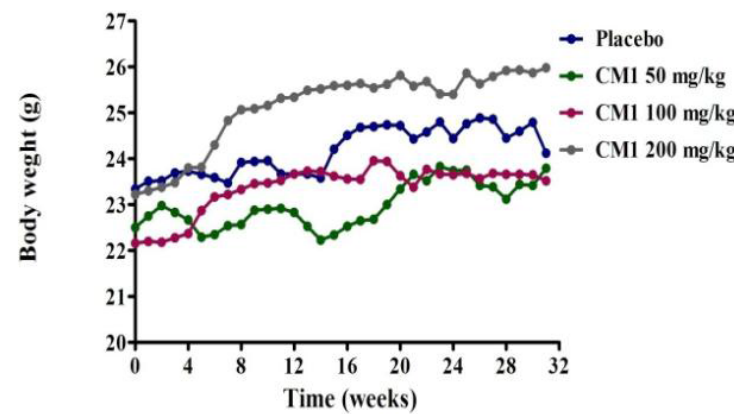

Female/N diet

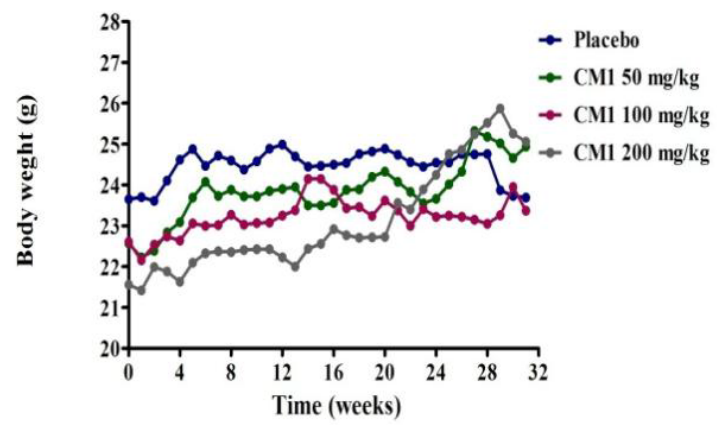

Female/Fe diet

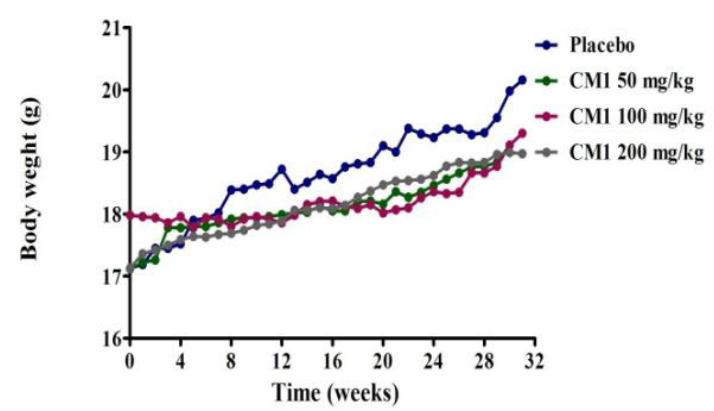

Figure 1: Changes in body weight (average values) of male and female $\beta$-thalassemia (BKO) mice fed with $\mathrm{N}$ and Fe diets and treated with $\mathrm{CM} 1$ (0-200 mg/kg) for 8 months. 
Citation: Chansiw N, Pangjit K, Phisalaphong C, Fucharoen S, Evans P, et al. (2013) Toxicity Study of a Novel Oral Iron Chelator: 1-(N-Acetyl-6Aminohexyl)-3 Hydroxy-2-Methylpyridin-4-One (CM1) in Transgenic ß-Thalassemia Mice. Vitam Miner 2: 116.

Page 5 of 6

\section{Discussion}

Iron is vital to cells, but an excess of iron is toxic. The genetic disorder, ß-thalassemia, is a secondary iron overload disease that requires repeated blood transfusions [15], and shows abnormal intestinal iron absorption. Iron is able to accumulate in many tissues and organs leading to tissue damage and organ dysfunction [16]. One way to remove excess iron is through iron chelation therapy. Nowadays, there are several widely used iron chelators that are available for iron chelation therapy. However, these compounds still have many adverse effects [17]. CM1 is a bidentrate synthetic oral iron chelator that is an analogue of DFP. In the previous work, we found that CM1 was an effective iron chelator. It can chelate iron efficiently in both in vitro and in vivo studies, but its toxicity is unknown. In this work, we investigated the toxicity of CM1 in transgenic $ß$-thalassemia mice under normal and iron overload conditions. The results indicated that there was no sign of toxicity under the clinical observation and there was no effect of CM1 on body weight under both conditions (Figure 1 and 2). Hemoglobin levels were not significantly different between the placebo and the treated groups, and this was similar in terms of the white blood cells and the platelet numbers (Table 1 and 3). The activity of AST, ALT and ALP slightly increased after 60 days for all groups and peaked on day 150 , especially among mice fed with an iron containing diet, but there was still no difference between the treated groups and the placebo groups. Moreover, the enzyme activities were observed to react in a dose-independent manner. In the pathological

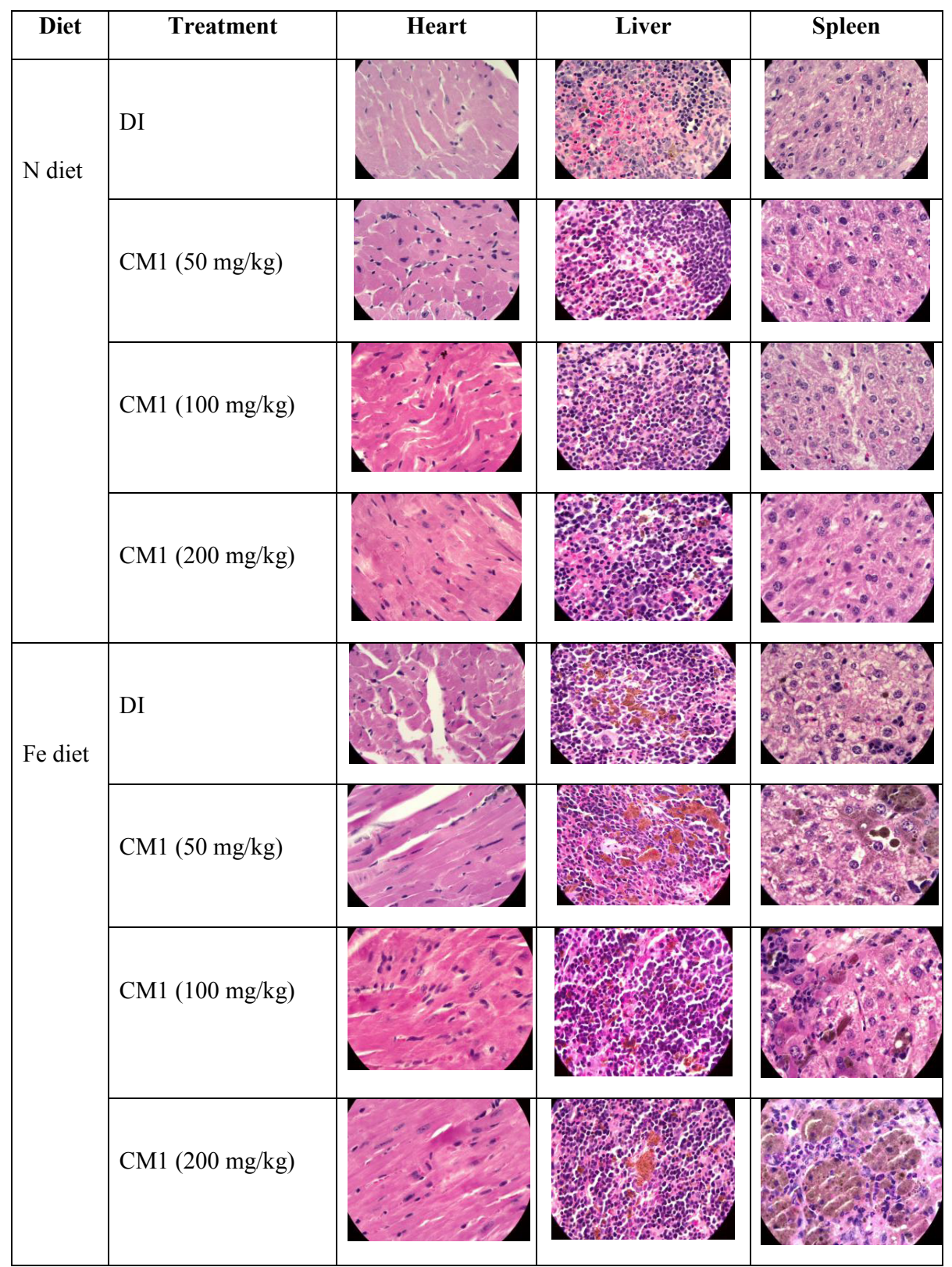

Figure 2: H\&E stained heart, liver and spleen tissues of male and female $\beta$-thalassemia (BKO) mice fed with $\mathrm{N}$ and Fe diets and treated with $\mathrm{CM} 1$ (0-200 mg/kg) for 8 months. 
Citation: Chansiw N, Pangjit K, Phisalaphong C, Fucharoen S, Evans P, et al. (2013) Toxicity Study of a Novel Oral Iron Chelator: 1-(N-Acetyl-6Aminohexyl)-3 Hydroxy-2-Methylpyridin-4-One (CM1) in Transgenic ß-Thalassemia Mice. Vitam Miner 2: 116.

examination, no significant pathological changes were observed in the hearts of the mice that received all doses of both feeding conditions, but their livers were still predominantly composed of neutrophil and lymphocytes. The spleen presented numerous scattered hemosiderinladen macrophage, especially among then iron loaded $B$-thalassemia mice. This is probably due to the liver being the major organ for iron storage. High iron accumulation induces liver damage, and thus, liver enzymes are released $[18,19]$. More parameters could not be investigated due to the limited blood volumes available in the mice.

\section{Conclusions}

CM1 is an effective iron chelator, and is not toxic to the peripheral blood and liver cells of $B$-thalassemia mice under normal and iron overload conditions. This may indicate that CM1 treatment is free from the severe side effects, such as leukopenia and thrombocytopenia.

\section{Acknowledgements}

This work was funded by the Royal Golden Jubilee PhD. Program, Thailand Research Fund; Faculty of Medicine Research Fund, Chiang Mai Univeristy, Thailand; and Research Chair Grant from the National Science and Technology Development Agency and Mahidol University through Professor Suthat Fucharoen, MD. We thank Institute of Research and Development, Government Pharmaceutical Organization for supplying CM1 chelator.

\section{References}

1. Andrews NC (2000) Iron metabolism: Iron deficiency and iron overload. Annu Rev Genomics Hum Genet 1: 75-98.

2. Taher A, Ismaeel H, Cappellini MD (2006) Thalassemia intermedia: revisited Blood Cells Mol Dis 37: 12-20.

3. Gutteridge JM, Halliwell B (1989) Iron toxicity and oxygen radicals. Baillieres Clin Haematol 2: 195-256.

4. Grootveld M, Blake DR, Merry P (1990) Iron overload, free radical damage, and rhesus haemolytic disease. Lancet 335: 1530-1531.

5. Younes M, Sause C, Siegers CP, Lemoine R (1988) Effect of deferrioxamine and diethyldithiocarbamate on paracetamol-induced hepato-and nephrotoxicity: The role of lipid peroxidation. J Appl Toxicol 8: 261-265.

6. Miyazawa K, Ohyashiki K, Urabe A, Hata T, Nakao S, et al. (2008) A safety, pharmacokinetic and pharmacodynamic investigation of deferasirox (Exjade,
ICL670) in patients with transfusion-dependent anemias and iron-overload: A Phase I study in Japan. Int J Hematol 88: 73-81

7. Wong A, Alder V, Robertson D, Papadimitriou J, Maserei J, et al. (1997) Live iron depletion and toxicity of the iron chelator deferiprone (L1, CP20) in the guinea pig. Biometals 10: 247-256.

8. Srichairatanakool S, Pangjit K, Phisalaphong C, Fucharoen S (2013) Evaluation of a novel oral iron chelator 1-(N-acetyl-6-aminohexyl)-3-hydroxypyridin-4-one (CM1) for treatment of iron overload in mice. Scientific Research 4: 153-163.

9. PangjitK, Banjerdpongchai R., Phisalaphong, C, Fucharoen S, Srichairatanakoo $\mathrm{S}$ (2012) Efficacy of 1-(N-acetyl-6-aminohexyl)-3-hydroxypyridin-4-one (CM1) in treatment of iron-loaded hepatocyte cultures. Scientific Research 3: 10601067.

10. Thephinlap C, Phisalaphong C, Lailerd N, Chattipakorn N, Winichagoon $P$, et al. (2011) Reversal of cardiac iron loading and dysfunction in thalassemic mice by curcuminoids. Med Chem 7: 62-69.

11. Srichairatanakool S, Pangjit K, Phisalaphong C (2009) Characterization and investigation of chelating ac-tivity of a novel iron chelator: 1-(N-acetyl-6aminohexyl) -3-hydroxypyridin-4-one. Thai patent No.0901000799.

12. Beal PJ, Cook LP, Lovric VA (1974) Drabkin's solution: A common source of error in haemoglobinometry. Pathology 6: 251-254.

13. Reitman S, Frankel S (1957) A colorimetric method for the determination of serum glutamic oxalacetic and glutamic pyruvic transaminases. Am J Clin Pathol 28: 56-63.

14. Tietz NW, Burtis CA, Duncan P, Ervin K, Petitclerc CJ, et al (1983) A reference method for measurement of alkaline phosphatase activity in human serum. Clin Chem 29: 751-761.

15. Ozment CP, Turi JL (2009) Iron overload following red blood cell transfusion and its impact on disease severity. Biochim Biophys Acta 1790: 694-701.

16. Taher A (2005) Iron overload in thalassemia and sickle cell disease. Semin Hematol 42: 5-9.

17. Chaston TB, Richardson DR (2003) Iron chelators for the treatment of iron overload disease: Relationship between structure, redox activity, and toxicity. Am J Hematol 73: 200-210.

18. Dhumeaux D, Hezode $C$ (2000) Iron overload and liver diseases (except fo hemochromatosis and dysmetabolic hepatosiderosis. Bull Acad Natl Med 184 349-354.

19. Thakerngpol K, Fucharoen S, Sumiyoshi A, Stitnimankarn T (1992) Liver tissue injury secondary to iron overload in beta-thalassemia/hemoglobin $E$ disease. Southeast Asian J Trop Med Public Health 23: 110-115. 